\title{
A focus on regulatory RNAs
}

\author{
RNA molecules are more than messengers between DNA and protein and exhibit rich regulatory functions in \\ development and disease. In this issue, we present a Focus on regulatory RNAs with specially commissioned \\ Review articles that discuss recent advances in this fast-growing area.
}

\begin{abstract}
n 1986, Walter Gilbert contemplated the idea of an 'RNA world' of early life forms that use only RNA molecules to carry genetic information and catalyse their own synthesis (Nature 319, 618 (1986)). Although DNA and protein have evolved to carry out these functions in complex cellular organisms, RNA molecules
\end{abstract} remain multifunctional. Aside from the classical mRNA, tRNA and rRNA that act between DNA and protein as central components in preparation for translation, numerous functional noncoding RNAs (ncRNAs) are transcribed from the genome. Some ncRNAs are capable of enzymatic catalysis during splicing and translation, whereas others control gene expression at various levels, constituting an RNAbased regulatory network. Small ncRNAs (sncRNAs), such as microRNAs (miRNAs), can suppress translation, degrade mRNA and epigenetically silence targeted loci. Next-generation sequencing has uncovered additional classes of sncRNAs that originate from small nucleolar RNA (snoRNA), tRNA or rRNA and act as miRNAs or modulate cellular processes such as ribosome biogenesis. Long ncRNAs (lncRNAs) are over 200 nucleotides in length and have highly diverse functions, which range from regulating gene expression at transcriptional and epigenetic levels in the nucleus to controlling mRNA turnover and translation in the cytosol. The compelling complexity of regulatory RNAs necessities a sophisticated surveillance system in which RNA processing enzymes work in concert with RNA editing and chemical modifications to precisely control RNA metabolism and functions. The enzymes that edit the RNA or write, erase and read chemical modifications are often linked to environmental signals. These interactions add another layer of complexity to the RNAbased regulatory network.

Many ncRNAs are expressed in a tissue-specific manner and regulate the development and physiology of complex organisms. Abnormal expression of miRNAs and lncRNAs has been linked to chronic illness, including cardiovascular disease, diabetes, neuronal degenerative disease and cancer. RNAi-based therapies targeting misregulated RNAs have been evaluated in clinical trials. Further illustrating their importance, a 2019 Breakthrough Prize in life sciences was awarded to Drs. Bennett and Krainer for developing antisense oligonucleotide therapy to treat children with spinal muscular atrophy (https://doi.org/10.1038/ d41586-018-07079-5). Additional encouraging initiatives came from the US Food and Drug Administration, who in 2018 approved the first siRNA-based drug, patisiran (Onpattro), to treat an adult peripheral nerve disease (https:// www.fda.gov/NewsEvents/Newsroom/ PressAnnouncements/ucm616518.htm).

In light of the fast growth and high interest of this discipline, we are delighted to present a Focus on regulatory RNAs, featuring two specially commissioned Review articles by Chen and co-authors, on 'Cellular functions of long noncoding RNAs' (Nat. Cell Biol. https://doi.org/10.1038/ s41556-019-0311-8), and Delaunay and Frye, on 'RNA modifications regulating cell fate in cancer' (Nat. Cell Biol. https://doi. org/10.1038/s41556-019-0319-0). The Focus can be found on a dedicated page (https:// www.nature.com/collections/hhaegiafea/) and includes an online library of related research articles and commentaries recently published in Nature Cell Biology.

In their Review, Chen and co-authors discuss the cellular functions of lncRNAs with a focus on how lncRNAs act in the nucleus. The authors summarize four major nuclear functions of lncRNAs, beginning with their regulatory role in genome organization through the modulation of chromatin compaction and the establishment of chromosomal contacts. lncRNAs also control the loading of histone or chromatin modifiers to chromatin, representing another function in chromatin remodelling. In addition to indirect effects on gene expression, lncRNAs can directly regulate transcription through formation of R-loops, interference with RNA polymerase machineries and transcription of the lncRNA locus. Chromatin is not the only territory in which lncRNAs are essential. The authors also discuss lncRNAs that act as architectural components or influence molecular processes inside nuclear bodies. Despite a preference for nuclear localisation, a subset of lncRNAs are also exported into the cytoplasm, where they regulate mRNA turnover, translation and protein modifications.

To date, more than 170 RNA modifications have been discovered, and the rapid deposition of these modifications enables quick adaption of cellular responses to environmental changes. Tumour cells dynamically adjust to changes in the microenvironment to ensure survival. RNA modifications in malignancies are the topic of the second Review article, written by Delaunay and Frye, which summarizes RNA modifications with emphasis on mRNA and tRNA modifications. $N^{6}$-Methyladenosine $\left(\mathrm{m}^{6} \mathrm{~A}\right)$ is the most abundant mRNA modification, regulating mRNA metabolism, including stability, splicing, and translation. tRNA modifications can be divided into two types, with modifications inside the anticodon loop determining protein synthesis speed and those outside of the loop controlling tRNA biogenesis. RNA modifications in tumour development are discussed in view of three pathological aspects they can influence: tumour-initiating cell fate, tumour invasion and metastasis and drug resistance. Further studies are required to dissect the precise contribution of RNAmodifying enzymes and their respective modifications to cancer, as well as to probe the potential crosstalk between different types of modifications.

Despite remarkable progress in delineating regulatory functions of RNA species, future research will continue to expand our knowledge of their complexity and implications in pathophysiological processes. At Nature Cell Biology, we await with interest what the future holds for this rapidly growing discipline and look forward to publishing forthcoming key discoveries involving regulatory RNAs. This Focus aims to provide a snapshot of current views, emerging trends and unanswered questions in this area. We thank our authors and referees for their contributions and hope that our readers will find the Focus informative and inspiring.

Published online: 2 May 2019 https://doi.org/10.1038/s41556-019-0325-2 